\title{
Surrogate Modeling of RF circuit blocks
}

\author{
Luciano De Tommasi ${ }^{1}$, Dirk Gorissen ${ }^{1}$, Jeroen $_{\text {Croon }}{ }^{2}$, and Tom Dhaene ${ }^{1}$ \\ 1 Ghent University - IBBT, Department of Information Technology (INTEC), \\ Gaston Crommenlaan 8 Bus 201, B-9050 Ghent, Belgium, \\ luciano.detommasi@ua.ac.be, \{dirk.gorissen, tom.dhaene\}@ugent.be. \\ 2 NXP-TSMC Research Center, High Tech Campus 37, PostBox WY4-01, \\ NL-5656AE Eindhoven, the Netherlands, jeroen.croon@nxp.com.
}

Surrogate models are a cost-effective replacement for expensive computer simulations in design space exploration. Literature has already demonstrated the feasibility of accurate surrogate models for single radio frequency (RF) and microwave devices. Within the European Marie Curie project O-MOORENICE! (Operational Model Order Reduction for Nanoscale IC Electronics) we aim to investigate the feasibility of the surrogate modeling approach for entire RF circuit blocks. This paper presents an overview about the surrogate model type selection problem for low noise amplifier modeling.

\section{Introduction}

Design space exploration of RF circuit blocks involves the solution of constrained multiobjective optimization problems in order to fulfill the performance specifications and perform what-if and sensitivity analysis. Optimization demands a large amount of circuit simulations so making the whole process very expensive.

We aim to develop scalable (parametrized) models of (non-linear) RF circuit blocks. This problem is too hard to be addressed applying model order reduction techniques. Furthermore, model order reduction is a model-driven approach: it needs the mathematical description of the system, which is not available when model equations are embedded into the circuit simulator. On the other hand, surrogate modeling is a data-driven approach, which does not make any analytical assumptions upon the model which has to be reduced. The simulator is seen as a 'black box' which accepts input samples and provides output samples, see Fig. 1. Basing upon such samples, a cheapto-evaluate surrogate model is trained. The surrogate has to be able to predict the outputs given by the simulator when a new input (which has not been used in the model training) is applied. This means that surrogate modeling is equivalent to construct the surface which fits the samples in Fig. 1 (in fact, surrogate models are also known as response surface models). 


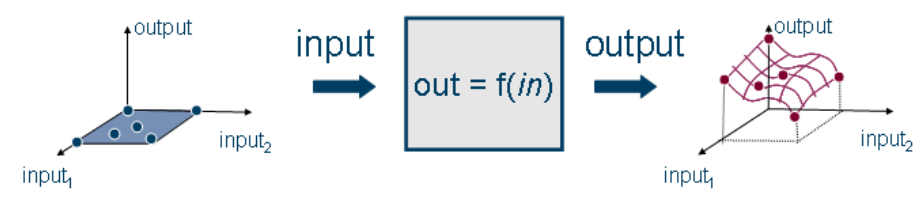

Fig. 1. Surrogate modeling approach.

Accurate surrogate models for single RF and microwave components have been already developed (e.g. using ANNs [1]). In this research activity, we aim to model complete RF circuit blocks. The first considered circuit block is a low noise amplifier (LNA) [2]. Other RF circuit blocks (e.g. mixers, VCOs, etc) can be analyzed following the same approach.

The behavior of an LNA is described by means of the admittance and noise functions, which are evaluated via accurate transistor-level simulations. Such functions are used to compute the performance figures (gain, input impedance, noise figure and power consumption) used by designers.

Each circuit simulation typically requires one to two minutes, which is a too long time to effectively explore how performance figures of LNA scale with key circuit-design parameters, such as the dimensions of transistors, passive components, signal properties and bias conditions. Therefore, the transistor level model can be usefully replaced with an accurate surrogate model (based on transistor level simulations) which is much cheaper to evaluate.

As first step towards an effective surrogate modeling process, the user of a modeling software environment has to find out which model type (among those ones available [3]) work better with his problem.

In this paper we summarize the results of a surrogate model type comparison for the LNA modeling problem.

\section{Software environment}

The surrogate modeling approach developed in this paper, is based on the SUrrogate MOdeling (SUMO) Matlab Toolbox [6]. The modeling flow is shown in Fig. 2. It is based on adaptive modeling and adaptive sampling loops.

The surrogate modeling process starts with the evaluation of an initial design (e.g. Latin hypercube, Box-Behnken, etc) which uniformly fills the design space (the number of samples is specified by the user). Based on this initial set of samples, one or more surrogate models are constructed. Adaptive modeling implies that a suitable optimization algorithm (e.g. hill climbing, particle swarm, genetic algorithm, DIRECT, etc) is used to tune relevant model hyperparameters, in order to minimize the error between model and data. Model error is evaluated according to one or more measures and functions. Afterwards, the models are ranked according to their score, and the best model is selected. 
In order to improve the accuracy, an adaptive sampling algorithm selects new samples based on the best performing models and the behavior of the reference function. In this work we applied the gradient-based method [6] because it has shown good performances with the LNA modeling problem.

After each sampling iteration, an adaptive modeling iteration including the new samples is started, and the whole process repeats itself until one of the following three conditions is satisfied: (1) the maximum allowed number of samples (specified by the user) has been reached, (2) the maximum allowed modeling time has been exceeded, or (3) the user required accuracy has been met.

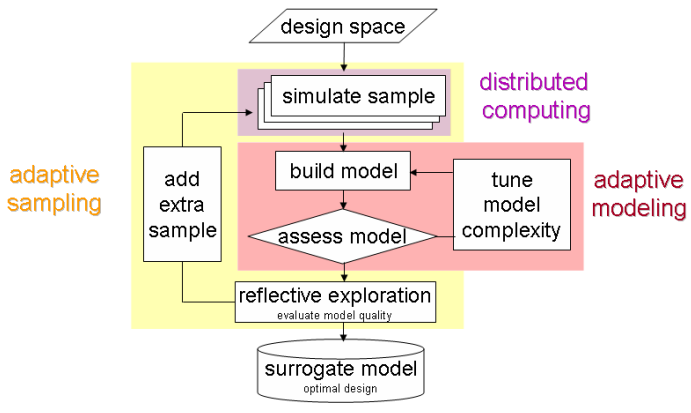

Fig. 2. Surrogate modeling flow.

\section{Surrogate model type selection}

Our feasibility study about surrogate modeling of low noise amplifiers aims to determine how many design variables can be included in a model and how many samples (number of simulations) are needed to generate the model.

A model is considered sufficiently accurate when its root relative square error is lower than 0.05. The maximum number of samples allowed is 1500 .

Several surrogate model types have been compared (as implemented in the SUMO Toolbox): artificial neural networks, rational functions, radial basis functions, least squares support vector machines and kriging [4],[5].

In order to reduce the computational cost of such comparison, transistor level simulations have been replaced with an analytical model of the $\mathrm{LNA}^{3}$ [4]. Although the accuracy of such analytical model is obviously not sufficient to replace the circuit simulator in the design process, it satisfactorily reproduces the shape of the simulator outputs.

Design parameters (input parameters) are, in order of importance: transistor width $W$, source inductance $L_{s}$, frequency $f$, transistor length $L$, gate

\footnotetext{
${ }^{3}$ Moreover, a cluster of PC has been used to build the surrogates.
} 
threshold voltage $V_{G T}$, gate series inductance $L_{m}$. The modeling software works with the following normalized parameters (characterized by the subscript ' $\mathrm{n}$ ') which lie in the interval [-1,1]: $W=100 \cdot 10^{-6} \cdot 10^{W_{n}} \mathrm{~m}, L_{s}=$ $0.5 \cdot 10^{-9} \cdot 10^{L_{s n}} H, f=\left(11+10 \cdot f_{n}\right) \cdot 10^{9} \mathrm{~Hz}, L=\left(90+30 \cdot L_{n}\right) \cdot 10^{-9} \mathrm{~m}$, $V_{G T}=0.275+0.2 \cdot V_{G T n} V, L_{m}=1 \cdot 10^{-9} \cdot 10^{L_{m n}} H$. As output parameters, we consider the admittances $y_{11}, y_{12}$, the input/output noise currents $\sqrt{\overline{i_{i n}^{2}}}$, $\sqrt{\overline{i_{\text {out }}^{2}}}$ and their correlation $\rho$. If a (normalized) input parameter is not taken into account in the modeling, it is clamped to 0 , the exception being $L$ which is clamped to -1 .

Examples of accurate surrogate models are shown in Figg. 3, 4 and 5.
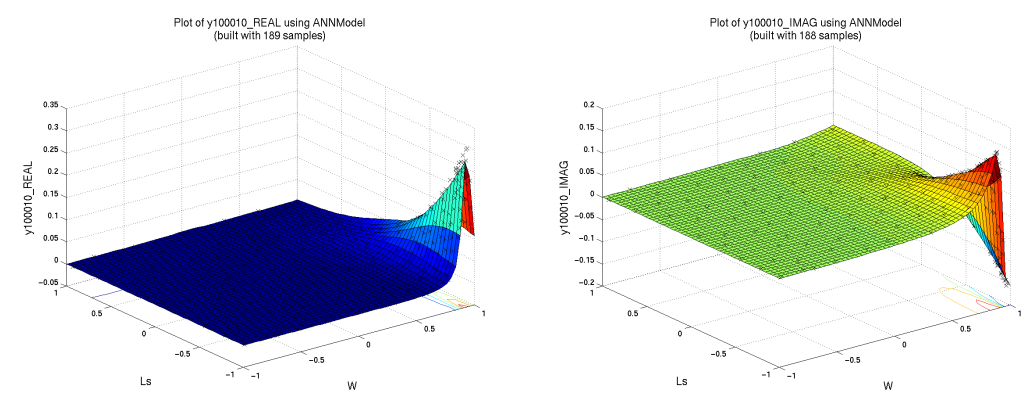

Fig. 3. ANN models of $y_{11}$ admittance with respect to inputs $W$ and $L_{s}$. Left: real part. Right: imaginary part.
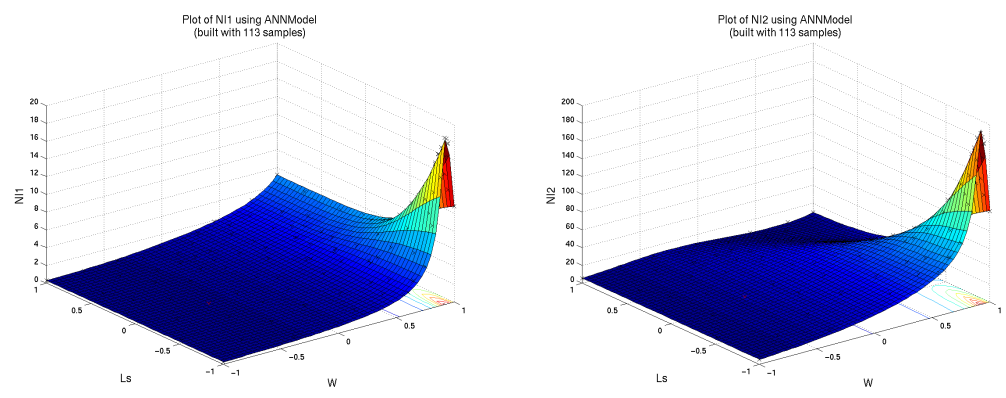

Fig. 4. ANN models of noise currents with respect to inputs $W$ and $L_{s}$. Left: input noise current. Right: output noise current. 

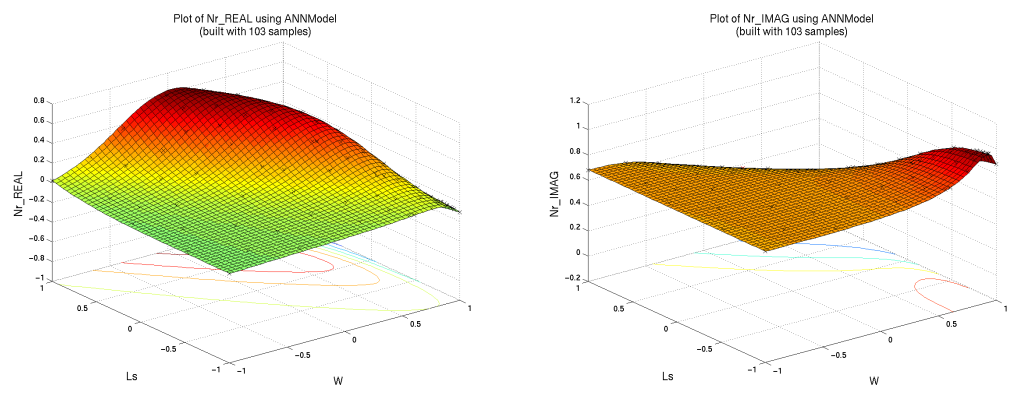

Fig. 5. ANN models of correlation between input and output noise currents with respect to inputs $W$ and $L_{s}$. Left: real part. Right: imaginary part.

Table 1 and Figg. 6 and 7 summarize the results ${ }^{4}$. Models which reach the accuracy level $R R S E<0.05$ are highlighted in bold. It is seen that accurate surrogate models of LNA can be obtained using at most four input parameters. Best model types are rational functions for admittances and ANNs for noise.

As future work, the best model types identified in this study will be used with transistor level simulations. In addition, further investigations will be aimed to improve the accuracy of models including five and six input parameters.
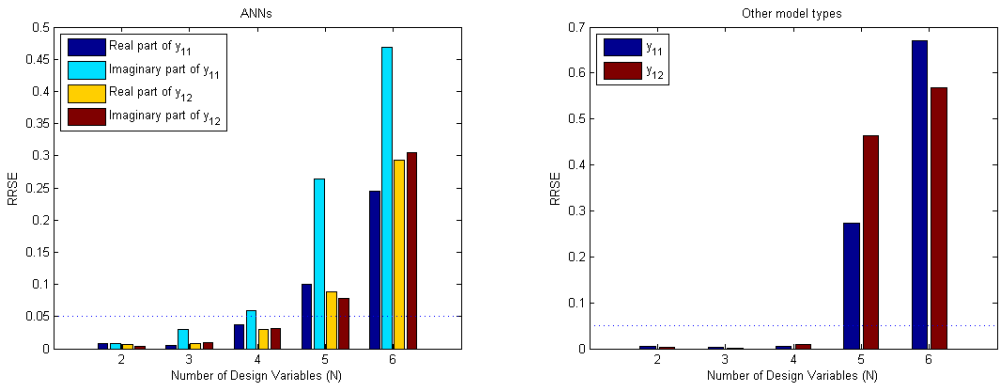

Fig. 6. Root relative square error of models of $y_{11}$ and $y_{12}$ as function of the number of design variables. Left: Artificial Neural Networks. Right: best model types other than ANN.

Acknowledgement. This work was supported by the European Commission through the Marie Curie Actions of its Sixth Program under the contract number MTKI-CT2006-042477. The authors thank Dr. Joost Rommes (NXP semiconductors, Eindhoven) for several stimulating discussions.

\footnotetext{
${ }^{4} \mathrm{HC}$ stands for 'hill climbing' optimization of hyperparameters [6]. GA stands for 'Genetic Algorithm' based optimization [8].
} 

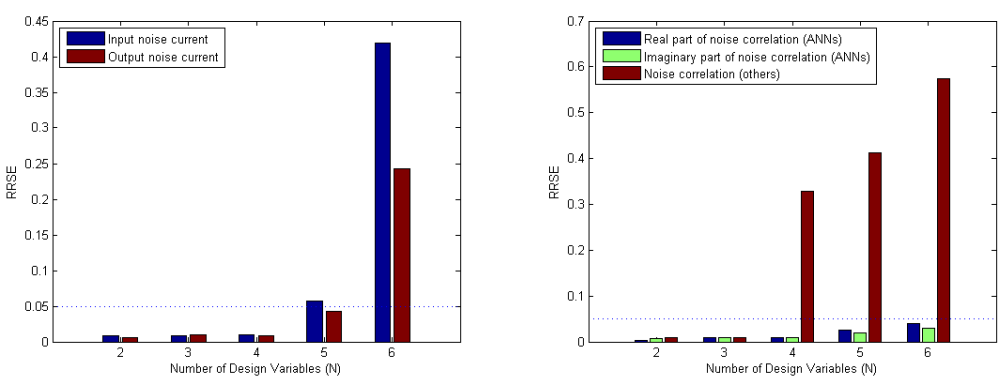

Fig. 7. Root relative square error of noise models as function of the number of design variables. Left: input and output noise currents (Artificial Neural Networks). Right: correlation between input and output currents.

Table 1. Best model type and hyperparameter optimization algoritms.

\begin{tabular}{|c|c|c|c|}
\hline $\mathrm{N} y_{11}$ & $y_{12}$ & input noise output noise & noise correlation \\
\hline 2 Rational HC & Rational GA & ANN GA Rational HC & ANN GA, Rational GA \\
\hline 3 Rational HC & Rational HC & ANN GA ANN GA & ANN GA, Rational HC \\
\hline 4 Rational GA & Rational GA & ANN GA ANN GA & ANN GA \\
\hline 5 Rational GA & RBF GA & ANN GA ANN GA & ANN GA \\
\hline $6 \mathrm{RBF}$ GA & RBF GA & ANN GA ANN GA & ANN GA \\
\hline
\end{tabular}

\section{References}

1. Zhang, Q.J., Gupta, K.C.: Neural Networks for RF and Microwave Design. Artech House, Boston - London (2000).

2. Lee, T.H.: The Design of CMOS Radio-Frequency Integrated Circuits (Second Edition). Cambridge University Press (2003).

3. Hendrickx, W., Gorissen, D., Dhaene, T.: Grid Enabled Sequential Design and Adaptive Metamodeling. In: Proceedings of the 2006 Winter Simulation Conference, WSC 2006, pp. 872-881. December 3-6 (2006).

4. Gorissen, D., De Tommasi, L., Croon, J., Dhaene, T.: Automatic Model Type Selection with Heterogeneous Evolution: An application to RF circuit block modeling. In: Proceedings of IEEE World Congress on Computational Intelligence, WCCI 2008, pp. 989-996. Hong Kong, China, June (2008).

5. Gorissen, D., De Tommasi, L., Crombecq, K., Dhaene, T.: Sequential Modeling of a Low Noise Amplifier with Neural Networks and Active Learning. Submitted to Neural Computing and Applications, (2008)

6. The SUrrogate MOdeling Toolbox Wiki Page. URL http://www.sumowiki.intec.ugent.be/.

7. Hendrickx, W., Dhaene, T.: Sequential design and rational metamodelling. In: Proceedings of the 2005 Winter Simulation Conference, WSC 2005, pp. 290-298. December 4-7 (2005)

8. The Mathworks: Genetic Algorithm and Direct Search Toolbox. URL http://www.mathworks.com/products/gads/. 
